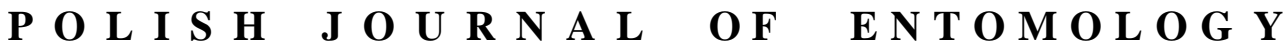

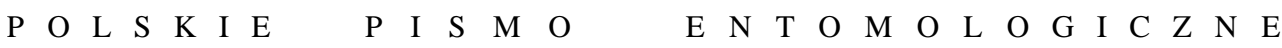

VOL. 85: $325-338$

Lublin

30 September 2016

DOI: $10.1515 /$ pjen-2016-0018

\section{New faunistic records of ground beetles (Coleoptera, Carabidae) from Hormozgan province, Iran}

\author{
SAEED AZAdBAKHSH ${ }^{*}$, AlinAGHI MiRMOAYEDI, SAMAD JAMALI \\ Department of Plant Protection of Razi University, Kermanshah, Iran
}

\begin{abstract}
This paper presents the results of a faunal study of ground beetles (Coleoptera: Carabidae) from Hormozgan province in southern Iran, which was carried out from winter 2015 to winter 2016. A total of 30 species belonging to 18 genera were collected and identified. Two species - Calodromius mayeti and Elaphropus (Tachyura) biblis - are reported from Iran for the first time; in addition, the occurrence of several species in Iran was confirmed.
\end{abstract}

KEY WORDS: Carabidae, Hormozgan province, southern Iran, faunistics.

\section{INTRODUCTION}

Hormozgan province, in southern Iran, lies on the northern shores of the Persian Gulf and Gulf of Oman. It has a hot and humid climate, with temperatures sometimes exceeding $49^{\circ} \mathrm{C}$ in summer and with very little precipitation all the year round. Almost all the mountains in the region are stony, dry and treeless. According to the first checklist of Iranian ground beetles, by AZADBAKHSH \& NOZARI (2015), 955 species of Carabidae are known from Iran. Although many papers on the ground beetles of Iran have been written over the years, most of these studies relate to the central or northern parts of Iran. The ground beetle fauna in the south of Iran is thus not well known.

In order to enhance our knowledge of the diversity of ground beetles in the south of Iran, we carried out surveys in Hormozgan province and studied the diversity of these insects in various ecosystems.

\footnotetext{
* Corresponding author: Azadbakhsh@live.com
} 


\section{Acknowledgemets}

Sincere thanks are due to Jacques CouLoN (France) for his help with identifying some of my specimens.

\section{MATERIALS AND METHODS}

The study material was collected in Hormozgan province, southern Iran, from winter 2015 to winter 2016. The material was gathered by hand from the ground, from under rocks or tree trunks, on river banks etc. The samples are preserved in an ethyl acetate atmosphere or in $96 \%$ alcohol. Identification is based on morphological characters and for this purpose we used the available keys (e.g. ARNDT et al. (2011), MiKHAILOV (1976), JABLOKOFFKHNZORIAN (1973), JUÑENT (2000)). Some material was identified on the basis of comparisons with identified specimens in the collections of one of the authors (S.A.).

All the material is deposited in S.A.'s personal collection. The list of species collected follows the nomenclature of LORENZ (2005); distribution notes follow LÖBL \& SMETANA (2003) and AZADBAKHSH \& NOZARI (2015).

\section{RESULTS AND DISCUSSION}

In this study, 30 species belonging to 18 genera are listed from Iran. The species Calodromius mayeti (BEDEL, 1907) and Elaphropus (Tachyura) biblis (BRITTON, 1948) are new national records.

\section{Subfamily Cicindelinae LATREILLE, 1802}

\section{Tribe Cicindelini LATREILLE, 1802}

\section{Cylindera (Eugrapha) pygmaea laetula (TSCHITSCHÉRINE, 1903)}

Material examined. 1 specimen, Hormozgan province, Minab road, 3.III.2016 $\left(27^{\circ} 19^{\prime} 31^{\prime \prime} \mathrm{N}, 56^{\circ} 28^{\prime} 9^{\prime \prime} \mathrm{E}\right),-11$ metres, S. AZADBAKHSH leg. et det; 3 specimens,

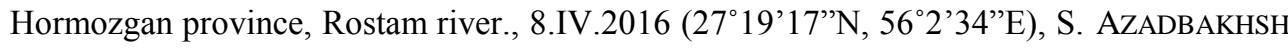
leg. et det.

Distribution. This subspecies was described from Bampur (Sistan va Baluchestan province) by TSCHITSCHÉRINE (1903) and recorded from IranShahr, $800 \mathrm{~m}$ (Sistan va Baluchestan province) by MANDL (1959) and Minab, Senderek and Jalabi (Hormozgan province) by NAVIAUX (1983).

Biology. This beetle was collected near saline water using a net. The habitat was very hot and humid. 


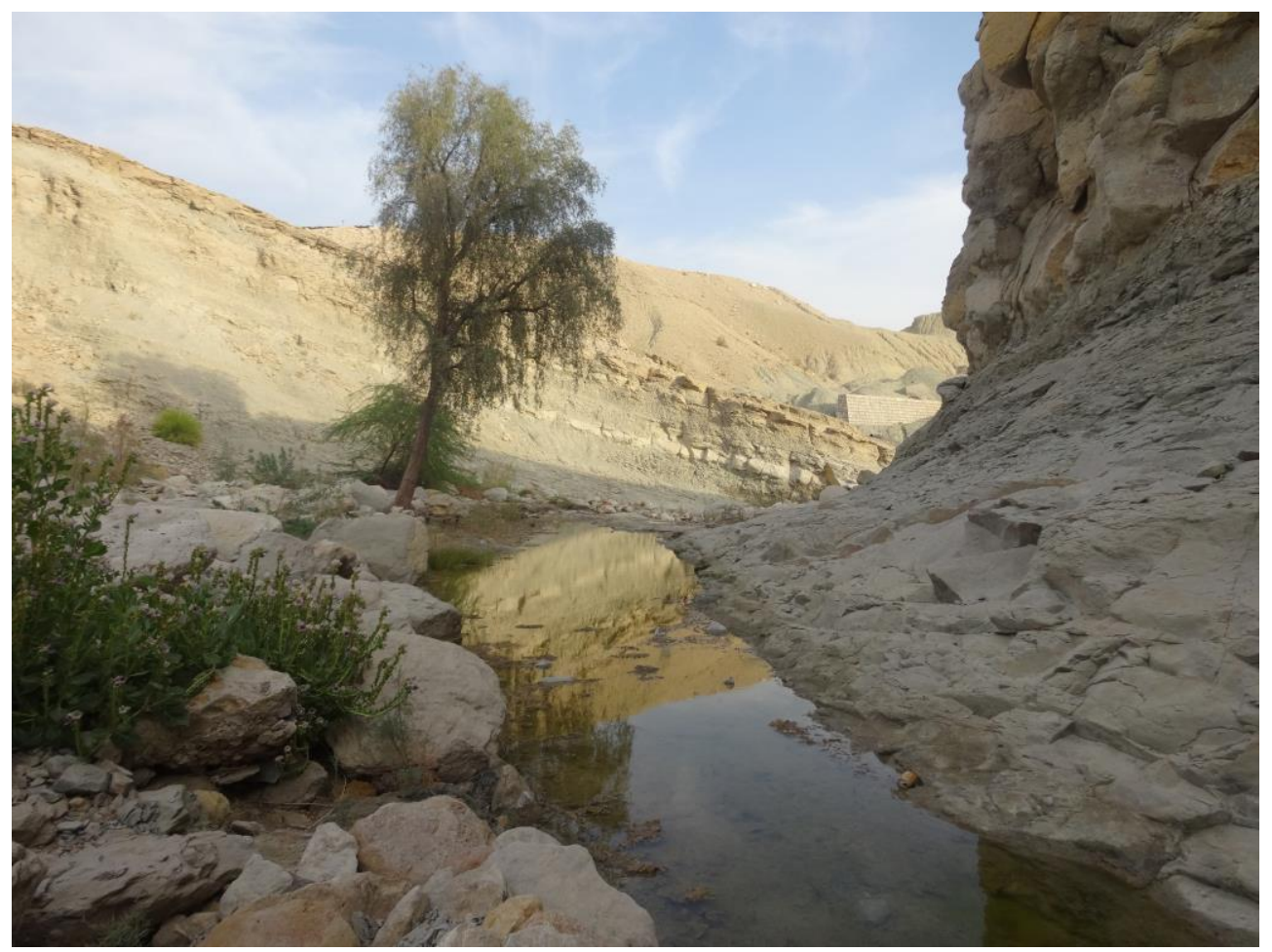

Fig. 1. Hormozgan province, Fin env., 13.IV.2016 (27 38'54’N, 5552’2’E), 300 metres; photo. S. AZADBAKHSH.

\section{Calomera diania TSCHITSCHÉRINE, 1903}

Material examined. 3 specimens, Hormozgan province, Fin env. (Fig. 1), 13.IV.2016 (27³8'54'N, 5552'2'E), 300 metres, S. AZADBAKHSH leg. et det; 1 specimen, Hormozgan province, Geno hot spring, 8.V.2015 (27 $26^{\prime} 45^{\prime}$ N, 56 $\left.17^{\prime} 54^{\prime \prime} \mathrm{E}\right)$, S. AZADBAKHSH leg. et det.

Distribution. This species was described from Duz-ab (Khuzestan province) by TSCHITSCHÉRINE (1903) and cited from a different region of Iran by AZADBAKHSH $\&$ NOZARI (2015).

Biology. It was collected near saline water using a net. The habitat was very hot and humid. 


\section{Calomera fischeri elongatosignata W. HORN, 1922}

Material examined. 2 specimens, Hormozgan province, Hasan-langi env., 18.III.2016 $\left(27^{\circ} 23^{\prime} 17^{\prime} \mathrm{N}, 56^{\circ} 53^{\prime} 2^{\prime \prime} \mathrm{E}\right) ; 3$ specimens, Hormozgan province, Bandar Abbas env.,

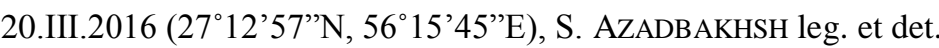

Distribution. This subspecies is distributed in the Arab Emirates, Afghanistan, Iran, Iraq, Kyrgyzstan, Kazakhstan, Oman, Pakistan, Tadzhikistan, Turkmenistan and Uzbekistan. In Iran, it was previously reported from Iranshar, (prov. Sistan va Baluchestan) and Anbar Abad (prov. Kerman) by MANDL (1959); Minab (prov. Hormozgan) by RivaliER (1967) and Dalaki (prov. Bushesehr), Bandar-e Shahpor "Khomeini" (prov. Khozestan), Bandar Abbas and Senderek (prov. Hormozgan), Delfar (prov. Kerman), Khoramabad (prov. Khoramabad), Daran (prov. Esfahan) and $37 \mathrm{~km} \mathrm{~N}$ of Iran ShahrKarvandar (prov. Sistan va Baluchestan) by NAVIAUX (1983).

Biology. Adults of this species were collected near fresh water. The habitat can be very different, however; my previous study showed that this species is very common in Hormozgan Province and can be found at any site with fresh or saline water, dry or with high humidity.

Cephalota (Taenidia) zarudniana zarudniana (TsCHITSCHÉRINE, 1903)

Material examined. 2 specimens, Hormozgan province, Geno env., 3.V.2015 $\left(27^{\circ} 26^{\prime} 45^{\prime}\right.$ N, $\left.56^{\circ} 17^{\prime} 54^{\prime \prime} \mathrm{E}\right)$, S. AZADBAKHSH leg. et det.

Distribution. This subspecies is distributed in Iran, Iraq, Israel, Saudi Arabia, Syria and Yemen. In Iran this species has been recorded in the south of the country. It was described by TSCHITSCHÉRINE (1903) from Neybandan (Fars province) and Kushah (Hormozgan province) and has been reported from Zabol (Sistan va Baluchestan province) by RIVALIER (1967), Niibandan (South Khorasan province) and Bandar Abbas (Hormozgan province) by MANDL (1976).

Biology. The specimens were collected on the bank of a river with saline/sulphurous water.

\section{Myriochila (Myriochila) melancholica melancholica (FABRICIUS, 1798)}

Material examined. 3 specimens, Hormozgan province, Hasan-langi env., 18.III.2016 $\left(27^{\circ} 23^{\prime} 17^{\prime}\right.$ 'N,56 53'2'"E); 1 specimen, Hormozgan province, Bandar Abbas env., 20.III.2016 (27 $12^{\circ} 57^{\prime} ’ \mathrm{~N}, 56^{\circ} 15^{\prime} 45^{\prime}$ 'E), S. AZADBAKHSH leg. et det.

Distribution. This subspecies is widely distributed in the Palaearctic region, mostly in Europe and the west of Asia. It has previously been reported from different parts of Iran. It is a new record for Hormozgan province.

Biology. The species was collected at both sites using a net near water. 


\section{Subfamily Carabinae LATREILLE, 1802}

Tribe Carabini LATREILLE, 1802

\section{Calosoma (Caminara) olivieri DEJEAN, 1831}

Material examined. 3 ex., Iran, Hormozgan Province, Fin env., 13.IV.2016

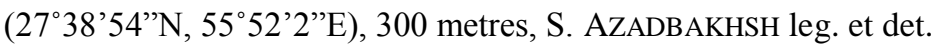

Distribution. This species is widely distributed in the Palaearctic region; in Iran it has been found in the south and east of the country (AZADBAKHSH \& NOZARI 2015).

Biology. The climate of Fin is dry and hot in summer with $5 \mathrm{~mm}$ rain in winter. Adults of this species were collected at night in light traps deployed near gardens.

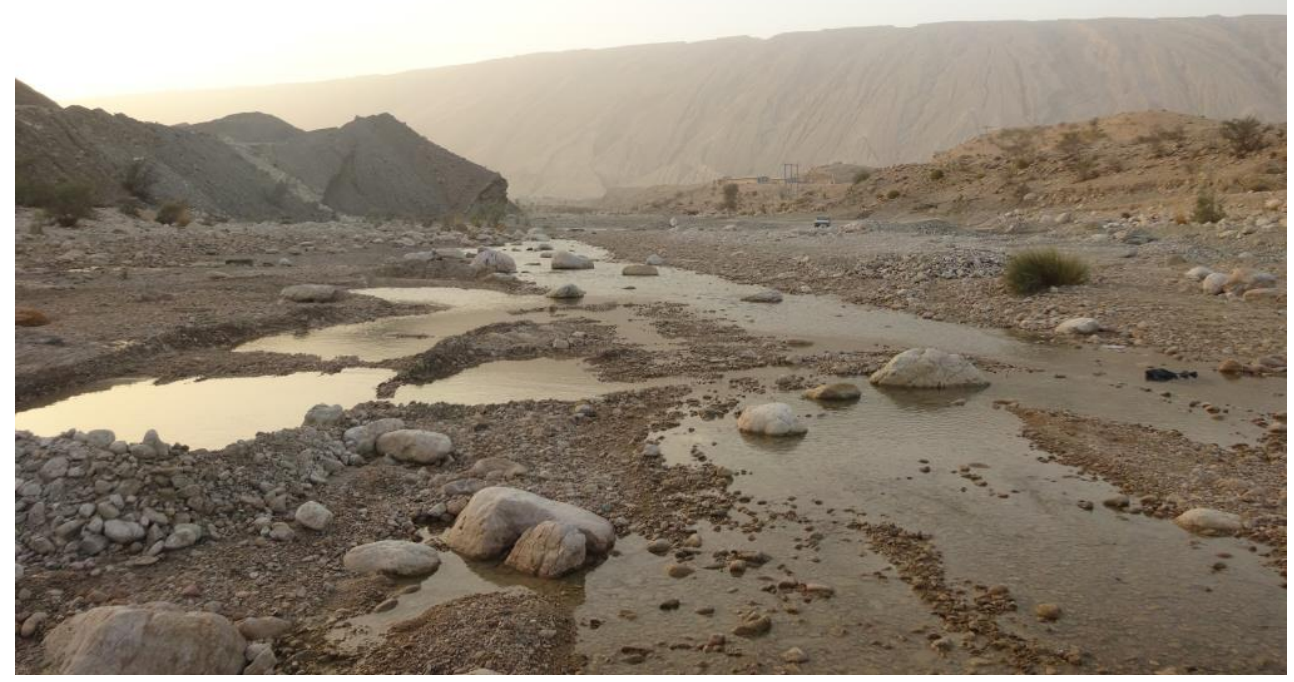

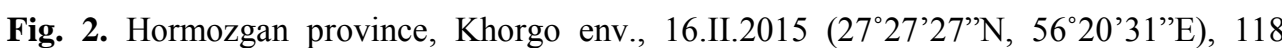
metres; photo. S. AZADBAKHSH. 


\section{Subfamily Scaritinae BONELLI, 1810}

Tribe Scaritini BONELLI, 1810

\section{Scarites (Parallelomorphus) subcylindricus CHAUdOIR, 1843}

Material examined. 7 specimens, Hormozgan province, Khorgo env. (Fig. 2), 16.II.2015 (27 27'27’N, 56 20'31'”E), 118 metres; 4 specimens, Hormozgan province,

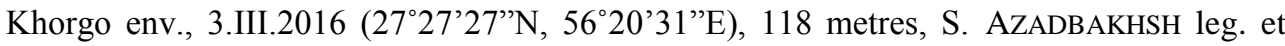
det.

Distribution. This species is distributed in Afghanistan, Algeria, Cyprus, Egypt (Sinai), Greece, Iran, India (Uttar Pradesh), Ireland, Israel, Kazakhstan, Libya, Pakistan, Saudi Arabia, Spain, Turkmenistan, Turkey and Yemen. In Iran, it was reported from Sabzawaran, Kerman province by MANDL (1963).

Biology. The specimens were collected by hand near a river bank under stones and mud.

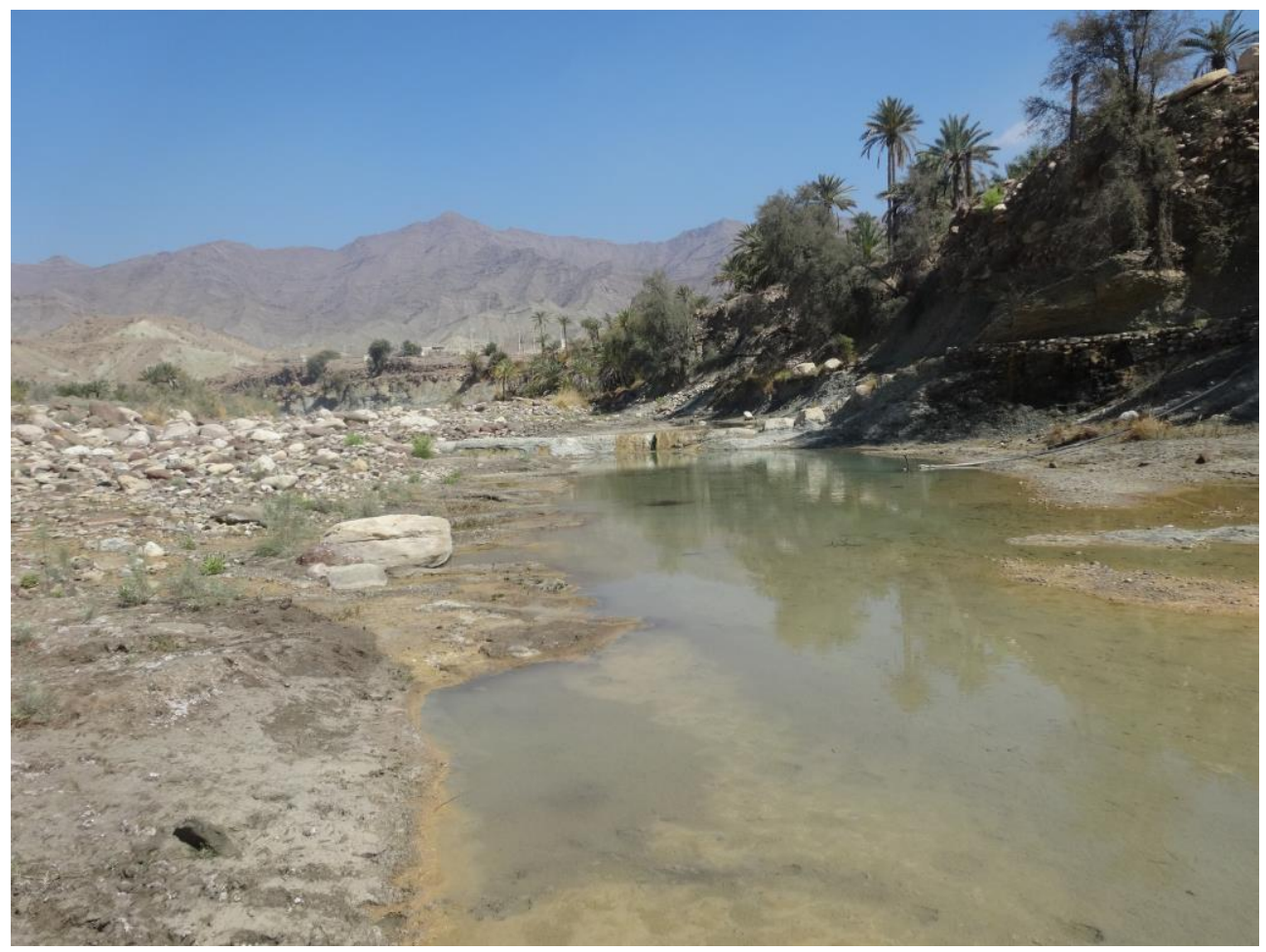

Fig. 3. Hormozgan province, Posht-Koh village, 21.III.2015 ( $\left.27^{\circ} 38^{\prime} 45^{\prime \prime} \mathrm{N}, 56^{\circ} 43^{\prime} 51^{\prime \prime} \mathrm{E}\right)$, 411 metres; photo. S. AZADBAKHSH. 


\section{Scarites (Scarites) procerus eurytus FISCHER VON WALDHEIM, 1828}

Material examined. 1 specimen, Hormozgan province, Posht-Koh village (Fig. 3), 21.III.2015 (27³8’45’N, 56 43’51’'E), 411 metres, S. AZADBAKHSH leg. et det.

Distribution. This subspecies is distributed in Afghanistan, Algeria, the Azores, Egypt, Georgia, Greece, Iran, Iraq, Italy, Kyrgyzstan, Kazakhstan, Libya, Morocco, Pakistan, Russia, Saudi Arabia, Spain, Syria, Turkmenistan, Turkey, Tunisia and Uzbekistan. It was reported from different parts of Iran by AZADBAKHSH \& NOZARI (2015).

Biology. It was collected by hand under a tree.

\section{Scarites (Parallelomorphus) terricola terricola BONELLI, 1813}

Material examined. 1 specimen, Iran, Hormozgan province, Ahmadi env., 20.II.2015 (275' 59’'N, 56 42’25’E), 932 metres, S. AZADBAKHSH let. \& det.

Distribution. This subspecies is distributed in Austria, the Azores, Bulgaria, China, Egypt, France, Greece, Hungary, Iran, Iraq, Italy, Kyrgyzstan, Kazakhstan, Libya, Moldavia, Mongolia, Morocco, Pakistan, Romania, Spain, Tadzhikistan, Turkmenistan, Turkey, Tunisia and Uzbekistan. In Iran, reported from Qazvin, Mazandaran, Gilan, Esfahan and Fars by AZADBAKHSH \& NOZARI (2015).

Biology. It was collected by hand near a river bank under a stone.

\section{Subfamily Trechinae BONELLI, 1810}

Tribe Trechini BONELLI, 1810

Perileptus (Pyrrotachys) jedlickai UENO, 1976

Material examined. 2 specimens, Hormozgan Province, Mand river, 12.II.2015 (27 30'26’N, 56 15’37'E), 201 metres; 1 specimen, Hormozgan Province, Roodan river, 23.II.2015 (27 $28^{\prime} 14^{\prime \prime} \mathrm{N}, 56^{\circ} 15^{\prime} 13^{\prime}$ 'E), 189 metres; 3 specimens, Hormozgan Province, Mola dam, 4.III.2015 (27²0’44”N, 56²'3”'E), 232 metres, S. AZADBAKHSH leg. et det.

Distribution. This species is distributed in Iran and Iraq. In Iran, it was recorded $13 \mathrm{~km}$ south-east of Nikshahr (prov. Sistan va Baluchestan) by MUILWIJK \& FELIX (2008).

Biology. Adults of this species were collected near both saline and freshwater river banks under stones. This species is very common in this region and is easily found on freshwater river banks.

\section{Perileptus (Perileptus) robustus JEANNEL, 1923}

Material examined. 1 specimen, Hormozgan Province, Posht-Koh village, 21.III.2015 $\left(27^{\circ} 38^{\prime} 45^{\prime} \mathrm{N}, 56^{\circ} 43^{\prime} 51^{\prime \prime} \mathrm{E}\right), 411$ metres; 4 specimens, Hormozgan Province, Mand river, 19.II.2015 (27 30’26’N, 56 15’37’'E), 185 metres, S. AZADBAKHSH leg. et det.

Distribution. This species is distributed in India and Iran. In Iran, it was recorded $13 \mathrm{~km}$ south-east of Nikshahr (prov. Sistan va Baluchestan) by MUILWIJK \& FELIX (2008). 
Biology. Adults of this species were collected near freshwater river banks under stones.

Tribe Bembidiini STEPHENS, 1827

Elaphropus (Tachyura) emeritus (PERINGUEY, 1898)

= biblis BRITTON, 1948

Material examined. 1 specimen, Hormozgan province, Roodan env., 3.VIII.2015 $\left(27^{\circ} 28^{\prime} 14^{\prime \prime} \mathrm{N}, 56^{\circ} 15^{\prime} 13^{\prime \prime} \mathrm{E}\right), 189$ metres, S. AZADBAKHSH leg. \& J. Coulon det., as biblis BRITTON, 1948.

Distribution. This species is distributed in Egypt (Sinai), Yemen and now in Iran. This is a new record for Iran.

Biology. An adult of this species was collected near a freshwater river bank under stones.

\section{Elaphropus (Tachyura) tetraspilus (SOLSKY, 1874)}

Material examined. 2 specimens, Hormozgan province, Ahmadi env., 19.II.2015 $\left(28^{\circ} 1^{\prime} 39^{\prime \prime} \mathrm{N}, 56^{\circ} 26^{\prime} 21^{\prime \prime} \mathrm{E}\right), 1121$ metres; 1 specimen, Hormozgan province, Shamil river, 6.III.2015, S. AZADBAKHSH leg. \& J. CoulON det.

Distribution. This species is distributed in Azerbaijan, Afghanistan, Georgia, Iran, India (Uttar Pradesh), Kyrgyzstan, Kazakhstan, Pakistan, Syria, Tadzhikistan, Turkmenistan and Uzbekistan. In Iran, it was reported from IranShahr (Sistan va Baluchestan province), Jiroft and Anbar-Abad (Kerman province) by KULT (1961).

Biology. Adults of this species were collected near freshwater river banks under stones.

\section{Elaphropus (Tachyura) euphratica (REITTER, 1885)}

Material examined. 2 specimens, Hormozgan province, Shamil env., 21.III.2015 ( $27^{\circ} 38^{\prime} 45^{\prime}$ 'N $\left.56^{\circ} 43^{\prime} 51^{\prime \prime} \mathrm{E}\right), 411$ metres; 1 specimen, Hormozgan province, Ahmadi env., 19.II.2015 $\left(27^{\circ} 55^{\prime} 22^{\prime} \mathrm{N}, 56^{\circ} 41^{\prime} 25^{\prime \prime} \mathrm{E}\right), 935$ metres; 4 specimens, Hormozgan province, Shamil env., 10.III.2015 (27 38'45”N, 56 43'51'E), 411 metres; 1 specimen, Hormozgan

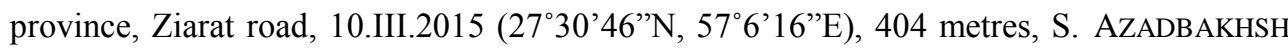
leg. \& J. Coulon det.

Distribution. This species is distributed in Azerbaijan, Georgia, Iran, Iraq, Israel, Kyrgyzstan, Kazakhstan, Pakistan, Syria, Tadzhikistan, Turkmenistan, Turkey and Uzbekistan. In Iran, it was reported from different parts of Iran by AZADBAKHSH \& NOZARI (2015).

Biology. Adults of this species were collected near freshwater river banks under stones. 


\section{Tachys (Tachys) angustulus REITTER, 1899}

Material examined. 4 specimens, Hormozgan province, Bandar Abbas env., Minab road, 10.III.2015 (27 19'31'N, 56 28'9'E), -11 metres; 1 specimen, Hormozgan province, Mand river, 12.II.2015 (27³6’15”N, 56 '1’32”), 289 metres, S. AZADBAKHSH leg. \& J. Coulon det.

Distribution. This species is distributed in Afghanistan, Iran, Kazakhstan, Turkmenistan, Tajikistan and Uzbekistan. In Iran, it was reported from Esfahan, Hormozgan, Gorgan and Khuzestan provinces by AZADBAKHSH \& NOZARI (2015).

Biology. Adults of this species were collected near freshwater river banks under stones.

\section{Tachys (Tachys) centralis J. SAHLBERG, 1900}

Material examined. 4 specimens, Hormozgan province, Bandar Abbas env., Minab road, 10.III.2015 (27 19'31'N, 56 28'9' E), -11 metres; 1 specimen, Hormozgan province, Ahmadi env., 19.II.2015 (28¹’39’N, 56²6’21’E), 1121 metres, S. AZADBAKHSH leg. \& J. Coulon det.

Distribution. This species is distributed in Kazakhstan, Turkmenistan, Tajikistan, Uzbekistan and Iran. In Iran, it was reported from Bonab and Marage (East Azerbaijan province) and Orumie (West Azerbaijan province) by COULON (2011).

Biology. Adults of this species were collected near freshwater river banks under stones.

\section{Subfamily Pterostichinae BonelLI, 1810}

Tribe Pterostichini BONELLI, 1810

Poecilus (Ancholeus) wollastoni (WOLLASTON, 1854)

Material examined. 1 specimen, Hormozgan province, Khorgo env., 16.II.2015 (2732'42”N, 56 26'57’'E), 168 metres, S. AZADBAKHSH leg. et det.

Distribution. This species is distributed in Algeria, the Canary Islands, Egypt, Iraq, Libya, Morocco, Madeira Arch., Saudi Arabia, Tunisia and Yemen. In Iran, it was reported from Kurusch-göl (East Azerbaijan province) by MANDL (1963) and Mahsha and Izeh lake (Khuzestan province) by WRASE (2009).

Biology. An adult of this species was collected near a freshwater river bank under stones.

\section{Subfamily Licininae BONELLI, 1810}

Tribe Chlaeniini BRULLÉ, 1834

Chlaenius (Chlaeniostenodes) canariensis persicus L. REDTENFOACHER, 1850

Material examined. 5 specimens, Hormozgan province, Fin env., 12.II.2015 $\left(27^{\circ} 38^{\prime} 34^{\prime \prime} \mathrm{N}, 5^{\circ} 53^{\prime} 58^{\prime \prime} \mathrm{E}\right), 330$ metres; 4 specimens, Hormozgan province, Posht-Koh village, 21.III.2015 (27 38’45”N, 56 43'51’E), 411 metres; 3 specimens, Iran, Hormozgan 
province, Khorgo env., 16.II.2015 (27³4'42”N, 56²6’57’E), 168 metres, S. AZADBAKHSH leg. et det.

Distribution. This subspecies is distributed in Afghanistan and Iran. In Iran, it has been found in the south of the country (AZADBAKHSH \& NOZARI, 2015).

Biology. Adults of this species were collected near freshwater river banks under stones.

\section{Chlaenius (Chlaenites) spoliatus spoliatus P. RossI, 1792}

Material examined. 3 specimens, Hormozgan province, Khorgo env., 20.II.2015 (27 $\left.34^{\circ} 42^{\prime \prime} \mathrm{N}, 56^{\circ} 26^{\prime} 57^{\prime \prime} \mathrm{E}\right), 168$ metres, S. AZADBAKHSH leg. et det.

Distribution. This subspecies has a wide distribution in the Palaearctic region. In Iran, it has been reported from Tehran, Chahar Mahal va Bakhtiari, Fars and Mazandaran provinces by AZADBAKHSH \& NOZARI (2015).

Biology. Adults of this species were collected near a freshwater river bank under stones.

\section{Chlaenius (Pseodochlaeniellus) puncticollis DEJEAN, 1826}

Material examined. 1 specimen, Hormozgan province, Ahmadi env., 20.II.2015 $\left(27^{\circ} 59^{\prime} 44^{\prime \prime} \mathrm{N}, 56^{\circ} 49^{\prime} 55^{\prime \prime} \mathrm{E}\right), 1084$ metres; 1 specimen, Hormozgan province, Geno env.,

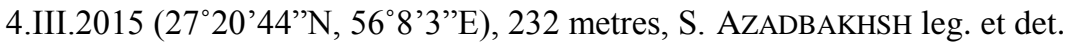

Distribution. This species is distributed in Iran, Afghanistan, Pakistan and India. In Iran, it has been found in the south of the country (AZADBAKHSH \& NOZARI 2015).

Biology. Adults of this species were collected near freshwater river banks under stones.

\section{Chlaenius (Stenochlaenius) dostojevskji TsCHITSCHÉRINE, 1895}

Material examined. 3 specimens, Hormozgan province, Sarzeh env., 12.II.2015 $\left(27^{\circ} 36^{\prime} 15^{\prime \prime} \mathrm{N}, 56^{\circ} 1^{\prime} 32^{\prime \prime} \mathrm{E}\right), 289$ metres; 3 specimens, Hormozgan province, Maroom env., 11.II.2015 (27 $\left.38^{\prime} 55^{\prime} \mathrm{N}, 55^{\circ} 52^{\prime} 3^{\prime \prime} \mathrm{E}\right), 320$ metres; 2 specimens, Hormozgan province, Khorgo env., 25.II.2015 (27 $\left.27^{\prime} 27^{\prime \prime} \mathrm{N}, 56^{\circ} 20^{\prime} 31^{\prime \prime} \mathrm{E}\right), 118$ metres, S. AZADBAKHSH leg. et det.

Distribution. This species is distributed in Afghanistan, Iran, Kashmir, Kazakhstan, Tadzhikistan and Uzbekistan. In Iran, it has been recorded in Kerman and Hormozgan provinces (AZADBAKHSH \& NOZARI 2015).

Biology. Adults of this species were collected near freshwater river banks under stones.

Chlaenius (Chlaeniellus) laeviplaga gambronicus AZADBAKHSH \& KIRSCHENHOFER, 2016

Material examined. 3 specimens, Hormozgan province, Sarzeh env., 12.II.2015 $\left(27^{\circ} 36^{\prime} 15^{\prime}\right.$ ”, $\left.56^{\circ} 1^{\prime} 32^{\prime \prime E}\right), 289$ metres; 3 specimens, Hormozgan province, Maroom env.,

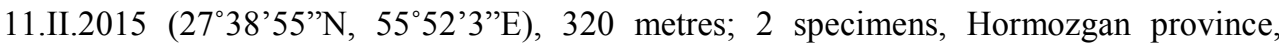


Khorgo env., 25.II.2015 (27 $\left.27^{\prime} 27^{\prime \prime} \mathrm{N}, 56^{\circ} 20^{\prime} 31^{\prime \prime} \mathrm{E}\right), 118$ metres, S. AZADBAKHSH leg. et det.

Distribution. This subspecies is distributed in southern Iran.

Biology. Adults of this species were collected near freshwater river banks under stones.

\section{Subfamily Harpalinae BONELLI, 1810}

Tribe Anisodactylini LACORDAIRE, 1854

Crasodactylus punctatus GUERIN-MENEVILLE, 1847

Material examined. 6 specimens, Hormozgan province, Deh-Barez env., 7.III.2015 $\left(27^{\circ} 27^{\prime} 2^{\prime \prime} \mathrm{N}, 57^{\circ} 9^{\prime} 27^{\prime \prime} \mathrm{E}\right), 205$ metres; 2 specimens, Hormozgan province, Posht-Koh village, 21.III.2015 ( $\left.27^{\circ} 38^{\prime} 45^{\prime \prime} \mathrm{N}, 56^{\circ} 43^{\prime} 51^{\prime \prime} \mathrm{E}\right), 411$ metres, S. AZADBAKHSH leg. et det.

Distribution. This species is distributed in Afghanistan, Algeria, Iran, Oman, Tunisia and Yemen. In Iran this species has been recorded in the south of the country.

Biology. Adults of this species were collected in light traps and under stones in gardens.

\section{Harpalus (Cryptophonus) tenebrosus DEJEAN, 1829}

Material examined. 4 specimens, Hormozgan province, Posht-Koh village, 21.III.2015 $\left(27^{\circ} 38^{\prime} 45^{\prime}\right.$ 'N , 56 $\left.43^{\circ} 51^{\prime \prime} \mathrm{E}\right), 411$ metres; 4 specimens, Hormozgan province, 16.IV.2015 (254' $\left.37^{\prime \prime} \mathrm{N}, 58^{\circ} 7^{\prime} 39^{\prime \prime} \mathrm{E}\right), 6$ metres, S. AZADBAKHSH leg. et det.

Distribution. This species is widely distributed in the Palaearctic region. In Iran it has been recorded from Mazandaran, Elburz, Tehran, Gilan, Fars, Khorasan, Kerman and East Azerbaijan by AZADBAKHSH \& NOZARI (2015).

Biology. Adults of this species were collected in light traps and under stones in gardens.

\section{Harpalus (Harpalus) metallinus metallinus MÉNÉTRIÉs, 1836}

Material examined. 10 specimens, Hormozgan province, Ahmadi env., 19.II.2015 (27 $55^{\prime} 22^{\prime}$ N, $56^{\circ} 41^{\prime} 25^{\prime}$ 'E), 935 metres, S. AZADBAKHSH leg. et det.

Distribution. This subspecies is distributed in Azerbaijan, Armenia, Bulgaria, Cyprus, Georgia, Greece, Iran, Iraq, Israel, Lebanon, Russia, Syria and Turkey. In Iran, it was reported from SABZAWARAN (Kerman province) by MANDL (1963) and Kermanshah by AZADBAKHSH \& NOZARI (2015).

Biology. Adults of this species were collected in light traps and under stones in gardens.

\section{Stenolophus (Egadroma) marginatus DEJEAN, 1829}

Material examined. 10 specimens, Hormozgan province, Posht-Koh village, 21.III.2015 $\left(27^{\circ} 38^{\prime} 45^{\prime} \mathrm{N}, 56^{\circ} 43^{\prime} 51^{\prime \prime} \mathrm{E}\right), 411$ metres; 1 specimen, Hormozgan province, Ahmadi env., 19.II.2015 (27 55’22”N, 56 41'25”E), 935 metres; 9 specimens, Hormozgan 
province, Ahmadi env., 19.II.2015 (28¹'39’N, 56²6’21’E), 1121 metres, S. AZADBAKHSH leg. et det.

Distribution. This species has a wide distribution in the Palaearctic region. In Iran, it has been reported from Hormozgan, Kerman and Tehran by AZADBAKHSH \& NOZARI (2015).

Biology. Adults of this species were collected near freshwater river banks under stones.

\section{Dixus eremita DEJEAN, 1825}

Material examined. 13 specimens, Hormozgan province, Posht-Koh village, 21.III.2015 (27³8’45’N, 56 43’51’'E), 411 metres, S. AZADBAKHSH leg. et det.

Distribution. This species is distributed in Azerbaijan, Albania, Armenia, Bulgaria, Cyprus, Greece, Iran, Iraq, Israel, Jordan, Kyrgyzstan, Kazakhstan, Macedonia, Moldavia, Romania, Russia, Syria, Tadzhikistan, Turkmenistan, Turkey, Ukraine and Uzbekistan. In Iran, it has been reported from Qazvin by AZADBAKHSH \& NOZARI (2015) and Sabzawaran (Kerman province) by MANDL (1963).

Biology. Adults of this species were collected under stones in gardens.

Subfamily Lebiinae BONELLI, 1810

Tribe Lebiini BONELLI, 1810

Calodromius mayeti (BEDEL, 1907)

Material examined. 2 specimens, Hormozgan province, Mand river, 12.II.2015 (27³0’26’N, 56 15’37’E), 201 metres, S. AZADBAKHSH leg. et det.

Distribution. This species is distributed in Morocco, Tunisia, the United Arab Emirates and now in Iran. It is a new record for Iran.

Biology. Adults of this species were collected in light traps.

\section{Masoreus (Masoreus) orientalis DEJEAN, 1828}

Material examined. 1 specimen, Hormozgan province, Bandar Abbas env., 20.III.2016 $\left(27^{\circ} 12^{\prime} 57^{\prime} \mathrm{N}, 56^{\circ} 15^{\prime} 45^{\prime \prime} \mathrm{E}\right)$, S. AZADBAKHSH leg. et det.

Distribution. This species is distributed in the Canary Islands, Egypt, Iran, India, Iraq, Madeira Arch., Saudi Arabia, Turkmenistan and Yemen. In Iran, it has been reported from Hormozgan province by AZADBAKHSH \& NOZARI (2015).

Biology. Adults of this species were collected in light traps.

\section{Lurestania gracilis ANICHTCHENKO, 2011}

Material examined. 1 specimen, Hormozgan province, Mand river, 12.II.2015 (2730’26’N, 56 15’37’'E), 201 metres, S. AZADBAKHSH leg. et det. 
Distribution. This species is distributed in southern and western Iran. It is a new record for the province.

Biology. An adult of this species was collected in a light trap.

\section{CONCLUSIONS}

In this study 30 species belonging to 18 genera of the family Carabidae are reported from Iran. Calodromius mayeti and Elaphropus (Tachyura) biblis are new records, reported from Iran for the first time. The species Lurestania gracilis was found for the second time in Iran; this record from southern Iran indicates that this species is distributed in the west and south of the country.

\section{REFERENCES}

Arndt E., Schnitter P., Sfenthourakis S., Wrase D.W. 2011. Ground Beetles (Carabidae) of Greece. Pensoft, Sofia.

AZADBAKhSh S., Nozari J. 2015. Checklist of the Iranian Ground Beetles (Coleoptera; Carabidae). Zootaxa 4024(1): 1-108.

Coulon J. 2011. Les Tachys du groupe scutellaris d'Europe occidentale et d'Afrique du Nord, et notes taxonomiques sur quelques autres espèces de Tachys (Coleoptera, Trechidae, Tachyini). Bulletin de la Société Entomologique de France 116(3): 303-320.

JABlokoff-Khnzorian S.M. 1973. Les Brachiniae du Caucase [Col. Carabidae]. Annales de la Société entomologique de France 9(8): 679-692.

JuÑENT S.R. 2000. The subtribes and genera of the Tribe Broscini (Coleoptera, Carabidae): Cladistic analysis, taxonomic treatment, and biogeographical considerations. Bulletin of the American Museum of Natural History 255: 1-90.

Kult K. 1961. Beitrag zur Kenntnis der Tachyiini aus Iran (Coleoptera, Carabidae). Stuttgarter Beiträge zur Naturkunde 57: 1-4.

LöBl I., Smetana A. 2003. Catalogue of Palaearctic Coleoptera. Volume I. Archostemata Myxophaga - Adephaga. Apollo Books, Stenstrup.

LORENZ W. 2005. Systematic list of extant ground beetles of the world (Coleoptera "Geadephaga": Trachypachidae and Carabidae incl. Paussinae, Cicindelinae, Rhysodinae). $2^{\text {nd }}$ Edition. Published by the author, Tutzing.

MANDL K. 1959. Eine Ausbeute an Cicindeliden aus Iran. Stuttgarter Beiträge zur Naturkunde 18 $1-3$.

MANDL K. 1963. Ergebnisse der Österreichischen Iran-Expedition 1949/50, Carabidae (Col.) Coleoptera IX. Teil. Sitzungsberichte der Akademie der Wissenschaften mathematischnaturwissenschaftliche Klasse 172, 179-192. 
MANDL K. 1976. Cicindela zarudniana probsti, eine neue Subspecies aus Persien (Cicindelidae, Col.). Zeitschrift der Arbeitsgemeinschaft Österreichischer Entomologen 28(1-3): 62-64.

Mikhailov V. 1976. Review of ground beetles of the genus Brachinus Weber (Coleoptera, Carabidae) from Middle Asia. Entomologicheskoe Obozrenie 55(2): 381-386. (in Russian)

Muilwijk J., Felix R. 2008. Description of three new species of the tribe Trechini (Col.: Carabidae) from south Iran. Journal of Entomological Society of Iran 28(1): 79-85.

NAviaux R. 1983. Coleoptera, Cicindelidae. Une approche de la faune d'Iran. Revue Scientifique du Bourbonnais 1983: 73-99.

Rivalier E. 1967. Contribution a la faune de l'Iran. 5. Coleoptères Cicindelidae. Annales de la société entomologique de France (N.S.) 3: 1099-1102.

TsCHITSCHÉRINE T. 1903. Mémoire sur les Cicindelidae des voyages de N. ZARUDNY dans la Perse orientale. Horae Societatis Entomologicae Rossicae 36: 1-21.

WRASE D.W. 2009. New or interesting records of Carabid beetles from Europe, Madeira, northern Africa, Turkey, from the Near East, Iran, Iraq, Kuwait, and Pakistan, with nomenclatorial and taxonomic notes (Coleoptera, Carabidae, Bembidiini, Brachinini, Cyclosomini, Elaphrini, Harpalini, Lebiini, Nebriini, Platynini, Pterostichini, Scaritini, Sphodrini, Zabrini). Linzer Biologische Beiträge 41(1): 901-935.

Received: 17 August 2015

Accepted: 1 October 2015 